\title{
Efficacy of Intrathecal MTX/Ara-C Combined with Systemic Chemotherapy in a Gastric Cancer Patient with Meningeal Carcinomatosis
}

\author{
Takahisa Yamasaki, Hirokazu Fukui, Hiroo Sei, Ken Hara, Hirotsugu Eda, Takashi Kondo, \\ Fumihiko Toyoshima, Tomoaki Kono, Katsuyuki Tozawa, Hisatomo Ikehara, \\ Toshihiko Tomita, Tadayuki Oshima, Jiro Watari and Hiroto Miwa
}

\begin{abstract}
A 35-year-old man was diagnosed to have gastric cancer by endoscopic and histological examinations. Staging laparoscopy detected peritoneal metastasis. Systemic chemotherapy was started, but the patient complained of severe headache. Subsequently, a lumbar puncture demonstrated adenocarcinoma cells in the spinal fluid, suggesting the occurrence of meningeal carcinomatosis (MC) from gastric cancer. MC occurs only rarely in patients with gastric cancer, but the prognosis is invariably poor. However, this patient nevertheless survived for 12 months after receiving intrathecal MTX/Ara-C together with systemic chemotherapy. Therefore, the early detection of meningeal irritation sign and intrathecal chemotherapy might greatly improve the prognosis of gastric cancer patients with MC.
\end{abstract}

Key words: gastric cancer, meningeal carcinomatosis, intrathecal chemotherapy, MTX, Ara-C

(Intern Med 55: 609-611, 2016)

(DOI: 10.2169/internalmedicine.55.5129)

\section{Introduction}

Meningeal carcinomatosis (MC), which is characterized by the diffuse infiltration of metastatic carcinoma to the meninges, occasionally accompanies breast or lung cancer, but rarely gastric cancer. The prognosis of patients with $\mathrm{MC}$ is invariably poor due to a typically late diagnosis and the lack of any effective treatment modalities. We herein report a case of MC in a patient with gastric cancer, who was diagnosed due to the onset of headache, which led us to identify the disease in a timely manner, and showed a prolonged survival after receiving intrathecal and systemic chemotherapy.

\section{Case Report}

A 35-year-old man who had suffered continuous epigastralgia was referred to our hospital. An endoscopic examination (Fig. 1A) revealed gastric cancer, and the diagnosis was confirmed by histology (Fig. 1B). Laboratory data showed no abnormalities including the level of CEA and CA19-9. Although contrast-enhanced CT demonstrated no metastatic lesions, staging laparoscopy detected peritoneal metastasis, both macroscopically (P1) and cytologically (CY1). Immunohistochemical examination of the cancer lesion confirmed that it was negative for HER2. Accordingly, we started systemic chemotherapy with $\mathrm{S} 1\left(80 \mathrm{mg} / \mathrm{m}^{2}\right.$, orally for 21 days $) /$ CDDP $\left(60 \mathrm{mg} / \mathrm{m}^{2}\right.$, intravenously on the eighth day), which is accepted as a first-line chemotherapy for unresectable stage IV gastric cancer in Japan (1). On the fifth day of chemotherapy, the patient first complained of severe headache, but he did not show any other neurological findings including paralysis, vomiting, nuchal stiffness, Kernig's sign or Brudzinski's sign. A gadolinium-enhanced magnetic resonance imaging (MRI) brain scan was performed, but this revealed nothing remarkable. Systemic chemotherapy was therefore routinely continued. However, since the patient had signs of meningeal irritation, we performed a lumbar punc- 

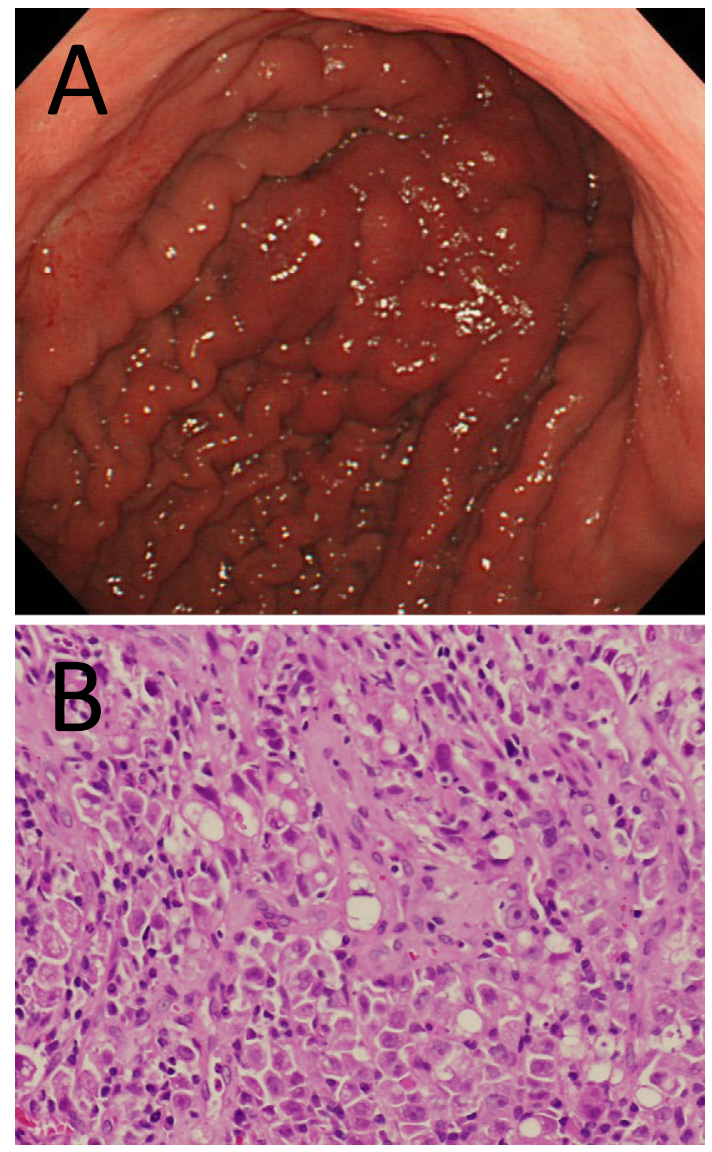

Figure 1. (A) An image taken by gastric endoscopy. The gastric folds at the greater curvature are thickened, and the findings are compatible with Borrmann type IV gastric cancer. (B) Histology of the gastric tumor. Poorly differentiated adenocarcinoma and signet-ring cell carcinoma cells show diffuse infiltration.

ture and a cytological examination demonstrated adenocarcinoma cells in the spinal fluid (Fig. 2), suggesting MC associated with the gastric cancer.

As no specific treatment has been established for meningeal carciomatosis in patients with gastric cancer, we planned intrathecal chemotherapy with MTX (10 mg)/Ara-C $(20 \mathrm{mg}) / \mathrm{PSL}(20 \mathrm{mg})$, which has been previously attempted in a few similar cases (2-4). After the first session of intrathecal chemotherapy, the patient's headache greatly improved, and the intrathecal pressure fell from 35 to $17 \mathrm{~mm}$ $\mathrm{H}_{2} \mathrm{O}$. This intrathecal chemotherapy was performed twice a week for the first two weeks, and then repeated once a week thereafter. The intrathecal chemotherapy was then performed continuously on an outpatient basis, and the spinal fluid at one point became negative for malignant cells. However, 11 months after the start of treatment, severe headache returned and leukoencephalopathy was diagnosed by MRI (Fig. 3). In addition, the level of tumor markers (CEA and CA19-9) began to increase, and chemotherapy-associated renal dysfunction developed. Although we changed the systemic chemotherapy from S1/CDDP to CPT-11 (100 mg/m ${ }^{2}$, intravenously every week), the patient died 12 months after the di-

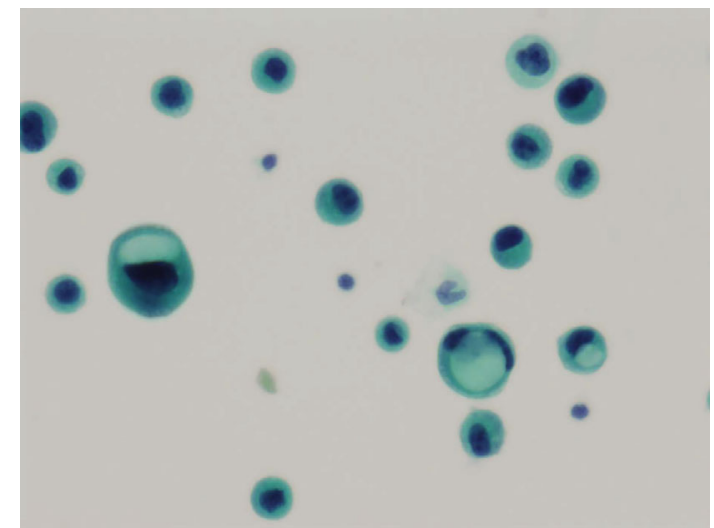

Figure 2. Cytology of the spinal fluid, showing floating adenocarcinoma cells with hyperchromatic nuclei. Some tumor cells have mucous vacuoles, resembling signet-ring cell carcinoma cells.

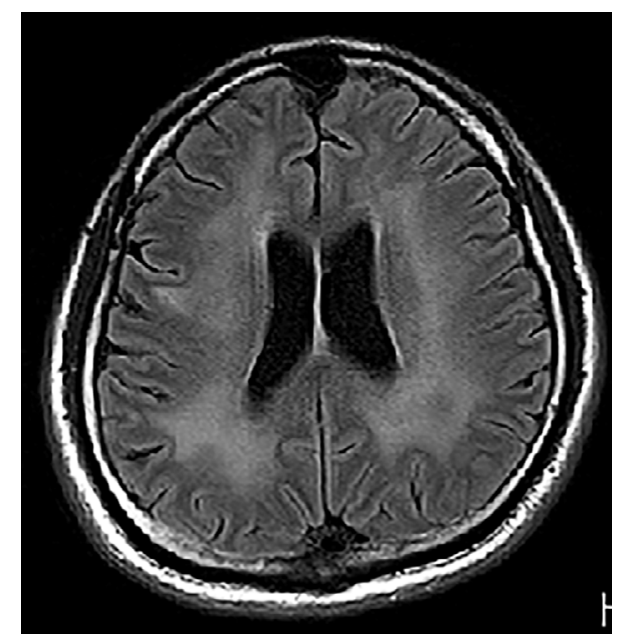

Figure 3. MRI showing the lateral ventricles to be surrounded by an abnormally enhanced signal.

agnosis of MC.

\section{Discussion}

Patients with cancer, especially those of the breast and lung, occasionally (approximately 5\%) also develop MC (5). However, MC occurs only rarely (0.16-0.69\%) in patients with gastric cancer $(2,6)$, and it is invariably associated with a dismal prognosis $(7,8)$. This may be due at least partly to a typically delayed diagnosis, and also due to the fact that imaging modalities such as CT or MRI often do not yield prognostically useful data $(9,10)$, as was the case in our present patient. However, since our patient continuously exhibited signs of meningeal irritation, we performed cytological testing of the spinal fluid and were thus able to confirm MC, suggesting that spinal fluid cytology rather than imaging examinations might be more useful to confirm the presence of MC.

The median survival of patients with MC is reported to be only four to six weeks without treatment, or four to six 
months even with therapy $(4,11,12)$. This poor survival may be due to not only the typically late diagnosis, but also due to the lack of any established treatments. Although systemic chemotherapy is an important strategy for the treatment of MC, anti-cancer drugs usually have difficulty in penetrating the blood-brain barrier. For this reason, wholebrain irradiation and intrathecal chemotherapy, each alone or in combination with systemic chemotherapy, have been tried for patients with $\mathrm{MC}(7,11,13-15)$. MTX plus AraC is reportedly better than MTX alone for intrathecal chemotherapy, in terms of outcome (16). Therefore, for the present patient, we combined intrathecal MTX/AraC with systemic chemotherapy. Although systemic and intrathecal chemotherapy are both palliative, they improved the symptoms of meningeal irritation, resulting in a better quality of life. Moreover, as the patient's MC was diagnosed early, he showed a longer survival (12 months) than the previously reported patients. On the other hand, although the intrathecal administration of anti-cancer drugs was effective, it may have been partly associated with the leukoencephalopathy that developed later, suggesting that there is still room for improvement in the selection of drugs for intrathecal chemotherapy and the timing of administration. Besides, intrathecal administration with a reservoir might be more effective to maintain the concentration of chemo-drugs at a high level, although we could not prepare it in this case due to clinical limitations. Advances in chemotherapy have considerably improved the prognosis of patients with unresectable gastric cancer. In this context, it is tempting to speculate that an increasing proportion of gastric cancer patients who survive for longer periods of time may develop rare patterns of metastasis at a late stage. Thus, as suggested in a recent work (17), we will encounter MC more frequently in the course of the treatment of gastric cancer patients in the future. We hope that the present report may be relevant when considering how to improve the treatment of $\mathrm{MC}$ in gastric cancer patients.

The authors state that they have no Conflict of Interest (COI).

\section{References}

1. Koizumi W, Narahara H, Hara T, et al. S-1 plus cisplatin versus S-
1 alone for first-line treatment of advanced gastric cancer (SPIRITS trial): a phase III trial. Lancet Oncol 9: 215-221, 2008.

2. Lee JL, Kang YK, Kim TW, et al. Leptomeningeal carcinomatosis in gastric cancer. J Neurooncol 66: 167-174, 2004.

3. Shapiro WR, Posner JB, Ushio Y, Chemik NL, Young DF. Treatment of meningeal neoplasms. Cancer Treat Rep 61: 733-743, 1977.

4. Wasserstrom WR, Glass JP, Posner JB. Diagnosis and treatment of leptomeningeal metastases from solid tumors: experience with 90 patients. Cancer 49: 759-772, 1982.

5. Yap HY, Yap BS, Tashima CK, DiStefano A, Blumenschein GR. Meningeal carcinomatosis in breast cancer. Cancer 42: 283-286, 1978.

6. Giglio P, Weinberg JS, Forman AD, Wolff R, Groves MD. Neoplastic meningitis in patients with adenocarcinoma of the gastrointestinal tract. Cancer 103: 2355-2362, 2005.

7. Bruno MK, Raizer J. Leptomeningeal metastases from solid tumors (meningeal carcinomatosis). Cancer Treat Res 125: 31-52, 2005.

8. DeAngelis LM, Boutros D. Leptomeningeal metastasis. Cancer Invest 23: 145-154, 2005.

9. Sze G, Soletsky S, Bronen R, Krol G. MR imaging of the cranial meninges with emphasis on contrast enhancement and meningeal carcinomatosis. AJNR Am J Neuroradiol 10: 965-975, 1989.

10. Straathof CS, de Bruin HG, Dippel DW, Vecht CJ. The diagnostic accuracy of magnetic resonance imaging and cerebrospinal fluid cytology in leptomeningeal metastasis. J Neurol 246: 810-814, 1999.

11. Chowdhary S, Chamberlain M. Leptomeningeal metastases: current concepts and management guidelines. J Natl Compr Canc Netw 3: 693-703, 2005.

12. Chamberlain MC, Kormanik PA, Barba D. Complications associated with intraventricular chemotherapy in patients with leptomeningeal metastases. J Neurosurg 87: 694-699, 1997.

13. Aiello-Laws L, Rutledge DN. Management of adult patients receiving intraventricular chemotherapy for the treatment of leptomeningeal metastasis. Clin J Oncol Nurs 12: 429-435, 2008.

14. Mehta M, Bradley K. Radiation therapy for leptomeningeal cancer. Cancer Treat Res 125: 147-158, 2005.

15. Taillibert S, Hildebrand J. Treatment of central nervous system metastases: parenchymal, epidural, and leptomeningeal. Curr Opin Oncol 18: 637-643, 2006.

16. Kim DY, Lee KW, Yun T, et al. Comparison of intrathecal chemotherapy for leptomeningeal carcinomatosis of a solid tumor: methotrexate alone versus methotrexate in combination with cytosine arabinoside and hydrocortisone. Jpn J Clin Oncol 33: 608612, 2003.

17. Emoto S, Ishigami H, Yamaguchi H, Yamashita H, Kaisaki S, Kiyatama J. Frequent development of leptomeningeal carcinomatosis in patients with peritoneal dissemination of gastric cancer. Gastric Cancer 14: 390-395, 2011.

(C) 2016 The Japanese Society of Internal Medicine http://www.naika.or.jp/imonline/index.html 